\title{
Characterization and Thermodynamic Stability of Polymorphs of Di(arylamino) Aryl Compound ASP3026
}

\author{
Kazuhiro Takeguchi, ${ }^{*, a, f}$ Yutaka Hirakura ${ }^{b}$ Kouji Yamazaki, ${ }^{c}$ Itsuro Shimada, ${ }^{d}$ Shigeru Ieda, ${ }^{e}$ \\ Minoru Okada, ${ }^{a}$ and Hiroshi Takiyama ${ }^{f}$ \\ ${ }^{a}$ Technology Process Chemistry Labs., Astellas Pharma Inc.; 160-2 Akahama, Takahagi, Ibaraki 318-0001, Japan: \\ ${ }^{b}$ Technology Analytical Research Labs., Astellas Pharma Inc.; 160-2 Akahama, Takahagi, Ibaraki 318-0001, Japan: \\ ${ }^{c}$ Drug Discovery Research Analysis \& Pharmacokinetics Research Labs., Astellas Pharma Inc.; 21 Miyukigaoka, \\ Tsukuba, Ibaraki 305-8585, Japan: ${ }^{d}$ Drug Discovery Research Medicinal Chemistry Research Labs., Astellas \\ Pharma Inc.; 21 Miyukigaoka, Tsukuba, Ibaraki 305-8585, Japan: ${ }^{e}$ Astellas Pharma Tech Co., Ltd.; 160-2 Akahama, \\ Takahagi, Ibaraki 318-0001, Japan: and ${ }^{f}$ Department of Chemical Engineering, Tokyo University of Agriculture and \\ Technology; 2-24-16 Naka-cho, Koganei, Tokyo 184-8588, Japan.
}

Received November 11, 2014; accepted March 23, 2015

ASP3026 ( $N$-\{2-methoxy-4-[4-(4-methylpiperazin-1-yl)piperidin-1-yl]phenyl\}- $N^{\prime}$-[2-(propane-2-sulfonyl)phenyl]-1,3,5-triazine-2,4-diamine) was developed in Astellas Pharma Inc. as a novel and selective inhibitor of the fusion protein EML4-ALK. We investigated the thermodynamic stability of five polymorphs of ASP3026 (A01, A02, A03, A04, and A05) in detail. To determine the most stable form at ambient temperature, powder X-ray diffraction, differential scanning calorimetry, and solubility measurements were conducted. Of the five polymorphs, A04 was the most stable and A05 was the least stable. The relationship between A04 and A03 and A04 and A01 were mutually monotropic, while that between A01 and A02 was enantiotropic. The transition temperature from A02 to A01 was estimated as $325 \mathrm{~K}$. A02 was more thermodynamically stable at ambient temperature than A01. Furthermore, the method to estimate polymorphic transition temperatures using solution calorimetry was found to be effective. The systematic characterization of ASP3026 polymorphs presented in this study enables the selective crystallization of the most stable form and design of solid formulations.

Key words polymorph characterization; transition temperature; solution calorimetry

ASP3026 ( $N$-\{2-methoxy-4-[4-(4-methylpiperazin-1-yl)piperidin1-yl]phenyl $\}-N^{\prime}$-[2-(propane-2-sulfonyl)phenyl]-1,3,5-triazine-2,4diamine) (Fig. 1) was developed as a novel and selective inhibitor of the fusion protein EML4-ALK. ${ }^{1)}$ In early studies, the active pharmaceutical ingredient (API) was obtained as a mixture of the polymorphs A01 and A02. However, despite attempted crystallization of these polymorphs, differences in specific manufacturing conditions induced further variations in the generated crystals.

To date, 5 polymorphs of ASP3026 (A01, A02, A03, A04, and A05) as well as a hydrate have been identified. ${ }^{2}{ }^{2} \mathrm{~A}$ polymorph is defined as a compound with multiple crystal structures due to the formation of different arrangements and conformations. Polymorphism is a widespread phenomenon observed in more than half of all APIs. ${ }^{3-5)}$ Polymorphs have different mechanical, thermal, physical, and chemical properties, such as melting point, density, dissolution rate, and solubility. Further, different polymorphs might have different bioavailabilities and stabilities. ${ }^{6)}$ Therefore, evaluation of the thermodynamic stability of polymorphs and control for not only API manufacturing but also drug development strategies is important. ${ }^{7)}$

In general, thermodynamic stability is evaluated using differential scanning calorimetry (DSC) data. When an endothermal solid-solid transition is observed during DSC analysis, the relationship between two polymorphs is enantiotropic and the transition temperature is below the endothermal temperature. When an exothermal solid-solid transition is observed during DSC analysis, the relationship between two polymorphs is monotropic and below the exothermal temperature (Heat of Transition Rule). ${ }^{8,9)}$ In cases where the polymorph with the higher melting point has a lower heat of fusion than the other polymorph, the relationship between two polymorphs is enantiotropic. When the polymorph with the higher melting point has a higher heat of fusion than the other polymorph, the relationship between two polymorphs is monotropic (Heat of Fusion Rule). ${ }^{8,9)}$

Selection of the polymorph which is most stable at ambient temperature is crucial to ensure that the drug is produced consistently both later in development and in the commercial manufacturing process. ${ }^{10,11)}$ One issue when determining the most stable polymorph at ambient temperature and when developing the selective crystallization process of the polymorph is determining the transition temperature when the relationship with the other polymorph is enantiotropic. In the present<smiles>COc1cc(N2CCC(N3CCN(C)CC3)CC2)ccc1Nc1ncnc(Nc2ccccc2S(=O)(=O)C(C)C)n1</smiles>

Fig. 1. Structure of ASP3026 
study, the most stable polymorph was determined at ambient temperature using powder X-ray diffraction (PXRD), DSC, and solubility measurement. The van't Hoff plot was used to determine transition temperatures. Another method derived from the van't Hoff equation was also introduced to determine the transition temperatures. The temperatures obtained were consistent, which gave credence to this latter method.

\section{Experimental}

Material ASP3026 was synthesized by Astellas Pharma Inc., and HPLC purity of the compound was above $97 \%$.

${ }^{1} \mathrm{H}-\mathrm{NMR} \quad\left(\mathrm{CDCL}_{3}, \quad 400 \mathrm{MHz}\right) \delta(\mathrm{ppm}): 1.31 \quad(\mathrm{~d}, \quad 6 \mathrm{H}$, $J=6.8 \mathrm{~Hz}), 1.58-1.80(\mathrm{~m}, 4 \mathrm{H}), 1.90-2.04(\mathrm{~m}, 2 \mathrm{H}), 2.16-2.84$ $(\mathrm{m}, 12 \mathrm{H}), 3.18-3.32(\mathrm{~m}, 1 \mathrm{H}), 3.66-3.76(\mathrm{~m}, 2 \mathrm{H}), 3.88(\mathrm{~s}$, $3 \mathrm{H}), 6.48-6.60(\mathrm{~m}, 2 \mathrm{H}), 7.18-7.26(\mathrm{~m}, 1 \mathrm{H}), 7.50-7.72(\mathrm{~m}$, $2 \mathrm{H}), 7.86-7.92(\mathrm{dd}, 1 \mathrm{H}, J=1.2,7.6 \mathrm{~Hz}), 8.06-8.16(\mathrm{~m}, 1 \mathrm{H})$, 8.28-8.48 (m, 1H), 8.48-8.62 (m, 1H), 9.28 (s, 1H).

Preparation of Polymorphs Each polymorph was obtained using various solvents and crystallization procedures. Solvents such as methanol, ethanol, isopropyl acetate, acetone, methyl ethyl ketone, acetonitrile were of special grade and purchased from Kanto Chemical Co., Inc. (Tokyo, Japan).

A01 ASP3026 was added to acetonitrile and dissolved at a reflux temperature. The solution was then rapidly cooled to ambient temperature, and the precipitated solid was obtained by filtration and drying.

A02 An aqueous solution of sodium chloride and sodium hydroxide was added to the methyl ethyl ketone solution of ASP3026 after reaction, and the organic layer was then separated and washed twice with sodium chloride solution. The organic layer was concentrated to switch methyl ethyl ketone to acetone, and the mixture was agitated at approximately $313 \mathrm{~K}$ and isopropyl acetate added at the same temperature. The mixture was then slowly cooled to approximately $273 \mathrm{~K}$, and the precipitated solid was obtained by filtration and drying.

A03 A02 was added to acetone, and the slurry was cooled to below $273 \mathrm{~K}$. A small quantity of A03 which was previously obtained by the same procedure was seeded, and the mixture was agitated for at least $3 \mathrm{~d}$. The precipitated solid was obtained by filtration and drying.

A04 A02 was added to 50\% acetone aqueous solvent preheated to approximately $333 \mathrm{~K}$. A small quantity of A04 which was previously obtained by the same procedure was seeded, and the mixture was agitated at the same temperature and cooled to approximately $298 \mathrm{~K}$. The precipitated solid was obtained by filtration and drying.

A05 ASP3026 was added to 50\% EtOH aqueous solvent and dissolved at approximately $353 \mathrm{~K}$. The solution was rapidly cooled to ambient temperature. A small quantity of A04 or A05 which was previously obtained by the same procedure was seeded, and the precipitated solid was obtained by filtration and drying.

Hydrate A05 was stored for approximately $7 \mathrm{~d}$ in a desiccator together with a beaker of water.

Characterization of Each Polymorph

PXRD ASP3026 polymorphs were evaluated using Miniflex (Rigaku Corp., Tokyo, Japan) under the following conditions: X-ray tube, copper; tube current, $15 \mathrm{~mA}$; tube voltage, $30 \mathrm{kV}$; sampling width, $0.010^{\circ}$; scanning speed, $2 \% \mathrm{~min}$; wavelength, $11.49 \mathrm{~mm}$; and range of measurement diffraction angles $(2 \theta), 3-35^{\circ}$.
DSC Analysis A TA Instruments Q-2000 calorimeter (TA Instruments, New Castle, DE, U.S.A.) was used to conduct DSC analysis under the following conditions: temperature range of measurement, ambient temperature to $220 \mathrm{~K}$ or higher; rate of temperature increase, $10 \mathrm{~K} / \mathrm{min}$; nitrogen flow rate, $50 \mathrm{~mL} / \mathrm{min}$; and sample pan material, aluminum.

Simultaneous XRD-DSC Measurement Simultaneous XRD-DSC measurement of A02 was conducted using RINT TTR II with Thermo Plus DSC attachment (Rigaku Corp.) under the following conditions: X-ray tube material, copper; tube current, $300 \mathrm{~mA}$; tube voltage, $50 \mathrm{kV}$; sampling width, $0.020^{\circ}$; scanning speed, $20 \% \mathrm{~min}$; wavelength: $1.54056 \AA$; range of measurement diffraction angles $(2 \theta), 5-30^{\circ}$; and heating rate, $2 \mathrm{~K} / \mathrm{min}$.

Solution Calorimetry The heat of solution for A01, A02, A03, and A04 was measured using Thermal Activity Monitor III (TA Instruments), at a measurement temperature of $298 \mathrm{~K}$. Approximately $20 \mathrm{mg}$ of each polymorph was dissolved in $100 \mathrm{~mL}$ of methyl ethyl ketone for calorimetry.

Solubility Measurement An excess quantity of each polymorph was added to the solvent. The temperature of the slurry was controlled with a thermostat bath, and the slurry was agitated for a predetermined period and then filtrated with a $0.45-\mu \mathrm{m}$ membrane filter. The concentration in the supernatant was measured via HPLC (Shimadzu Corp., Kyoto, Japan; or Hitachi High-Tech, Tokyo, Japan) under the following conditions: mobile phase, $0.1 \%$ perchloric acid aq. to acetonitrile $3: 1$; column, L-column 2 ODS $(4.6 \mathrm{~mm} \times 150 \mathrm{~mm}$, $5 \mu \mathrm{m}$, Chemical Evaluation and Research Institute), flow rate, $1 \mathrm{~mL} / \mathrm{min}$; column temperature, $313 \mathrm{~K}$; and detection, UV $225 \mathrm{~nm}$.

\section{Results and Discussion}

Powder X-Ray Diffraction (PXRD) The powder X-ray diffraction patterns of A01, A02, A03, A04, A05, and hydrate (Fig. 2) appeared to be mutually distinct, indicating the presence of six crystal structures. Hydrate was unstable in the organic solvents, therefore thermodynamic stability of hydrate was not evaluated in this study.

DSC Analysis and Simultaneous XRD-DSC Measurement The DSC thermograms of the five polymorphs are shown in Fig. 3.

-A01 showed an endothermic peak due to melting at approximately $433 \mathrm{~K}$. The heat of fusion of A01 was approximately $46 \mathrm{~kJ} / \mathrm{mol}$.

-A02 showed a small endothermic peak at approximately $409 \mathrm{~K}$ and a large one at approximately $433 \mathrm{~K}$, the latter of which was the same as that observed for A01. Figure 4 showed the simultaneous XRD-DSC data of A02. While the melting point of A02 was not observed in DSC analysis, a small endothermic peak was evident at approximately $409 \mathrm{~K}$ due to the solid phase transition from A02 to A01. According to the Heat of Transition Rule, the relationship between A01 and A02 is considered enantiotropic, and the transition temperature is below $409 \mathrm{~K}$. A02 may be more thermodynamically stable than A01 at ambient temperature.

-A04 exhibited an endothermic peak due to melting at approximately $453 \mathrm{~K}$. The heat of fusion of A04 was approximately $55 \mathrm{~kJ} / \mathrm{mol}$, and on comparing this value with that of A01, the relationship between A04 and A01 was deemed 


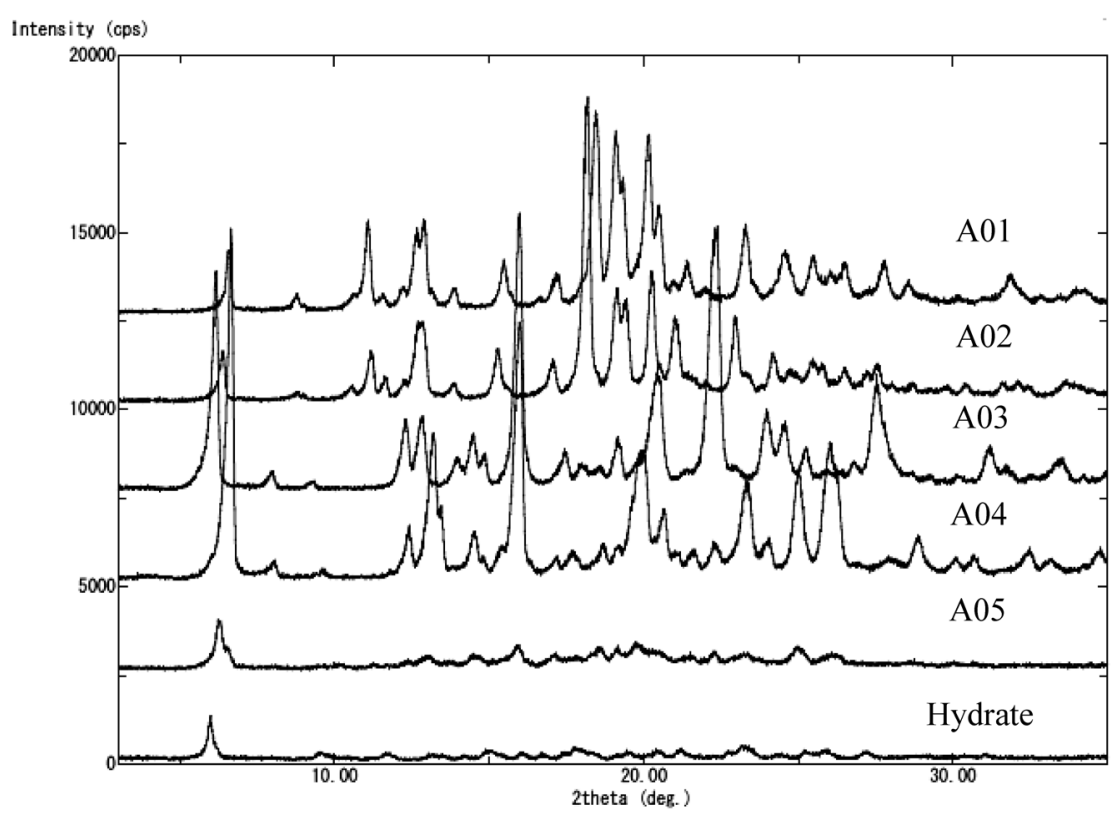

Fig. 2. X-Ray Powder Diffraction Patterns

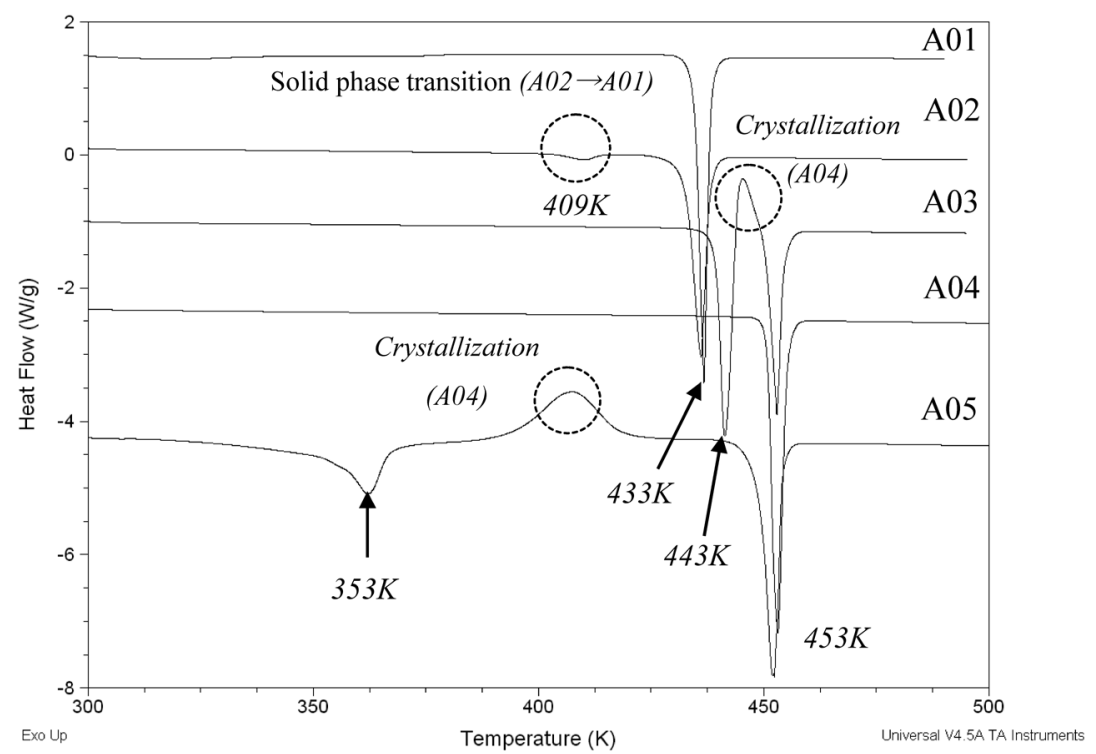

Fig. 3. DSC Thermograms

monotropic according to the Heat of Fusion Rule. A04 was thus considered to be more thermodynamically stable than A01 at ambient temperature.

-A05 exhibited an endothermic peak due to melting at approximately $353 \mathrm{~K}$. An exothermic peak likely due to crystallization of A04 was observed at approximately $413 \mathrm{~K}$, and on comparing this value with the heat of fusion for A01, the relationship between A05 and A01 was deemed monotropic according to the Heat of Fusion Rule. A01 was thus considered to be more thermodynamically stable than A05 at ambient temperature.

-A03 melted at approximately $443 \mathrm{~K}$, followed by crystallization of A04. The fact that crystallization of A04 occurred directly after melting of A03 obscured the heat of fusion of A03 and measurement of solubility was therefore required to determine the relationships between the thermodynamic stability of the polymorphs.
Solubility The solubility of each polymorph, evaluated with methyl ethyl ketone, is shown in Table 1. The van't Hoff plot of each polymorph is shown in Fig. 5. The solubility data indicate that A04 is the most stable of all five polymorphs at ambient temperature.

Solubility evaluation showed that the transition temperature from A03 to A04 was not lower than the melting point. The relationship between A03 and A04 was therefore deemed to be monotropic, cementing A04 as more stable than A03 at ambient temperature. The transition temperature from A04 to A03 was calculated as $1400 \mathrm{~K}$, which was markedly higher than the melting point, according to the van't Hoff plot. An equi-solubility temperature was found at $325 \mathrm{~K}$ between $\mathrm{A} 01$ and $\mathrm{A} 02$, indicating that these polymorphs are mutually enantiotropic, which is consistent with the above DSC findings. A02 is more stable than A01 at ambient temperature, also consistent with DSC findings. 


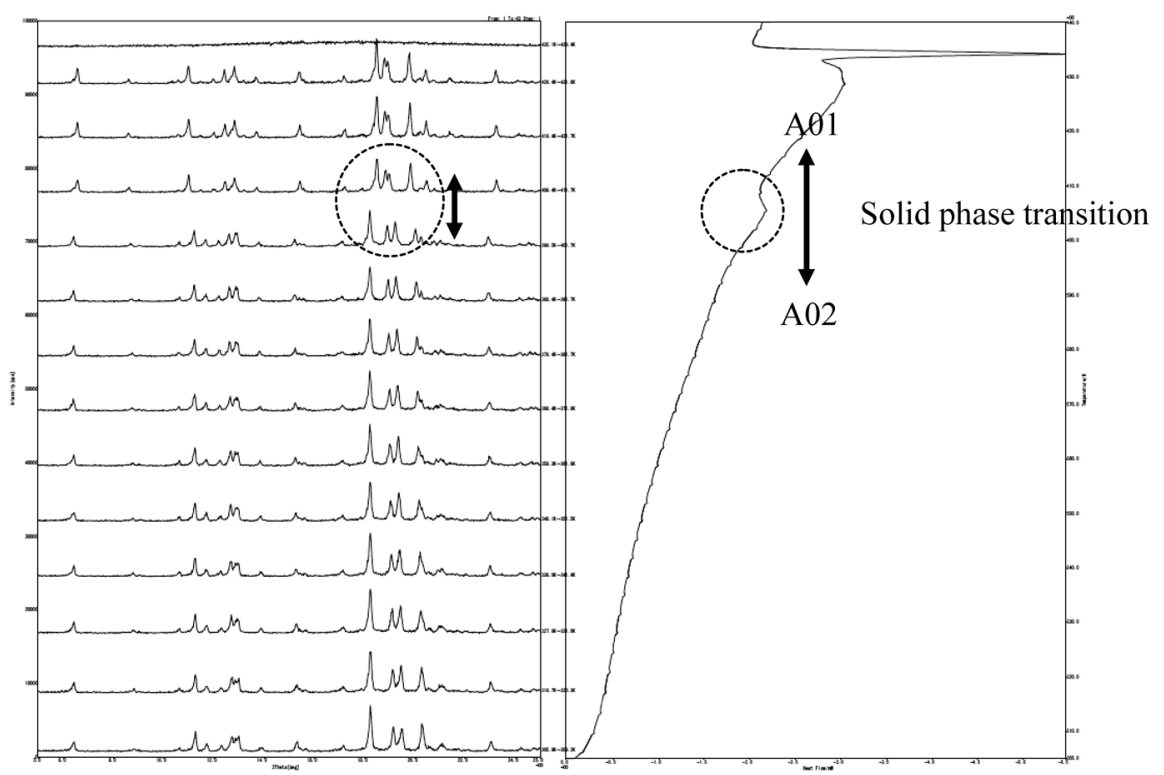

Fig. 4. Simultaneous XRD-DSC Data of A02

Table 1. Solubility in Methyl Ethyl Ketone

\begin{tabular}{ccccc}
\hline \hline \multirow{2}{*}{ Temperature } & \multicolumn{4}{c}{ Solubility $[\mathrm{mol} / \mathrm{mol}]$} \\
\cline { 2 - 5 } & A01 & A02 & A03 & A04 \\
\hline $298 \mathrm{~K}$ & 0.00221 & 0.00207 & 0.00109 & 0.00089 \\
$308 \mathrm{~K}$ & 0.00346 & 0.00342 & - & - \\
$313 \mathrm{~K}$ & 0.00449 & 0.00394 & 0.00186 & 0.00174 \\
$323 \mathrm{~K}$ & 0.00612 & 0.00623 & - & - \\
$333 \mathrm{~K}$ & 0.00977 & 0.01013 & 0.00446 & 0.00383 \\
$350 \mathrm{~K}$ & - & - & 0.00902 & 0.00790 \\
\hline
\end{tabular}

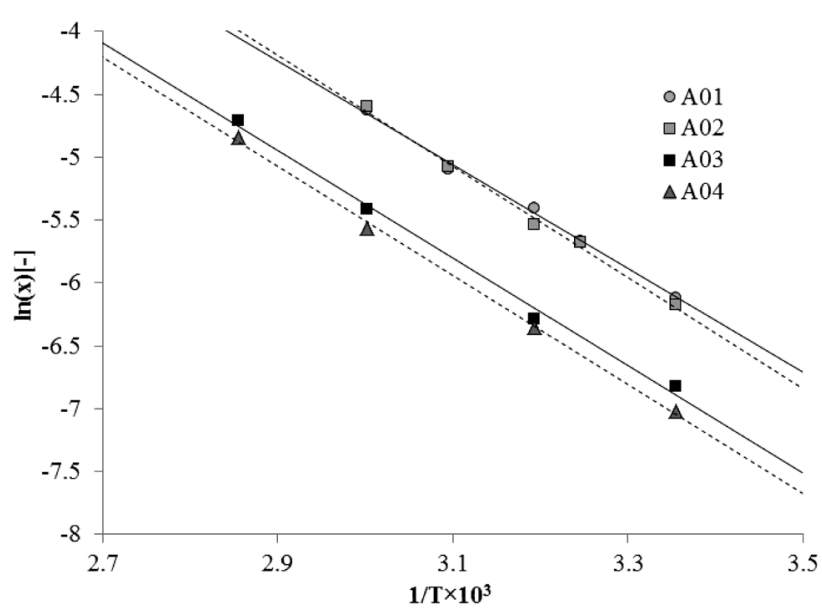

Fig. 5. van’t Hoff Plot

The solubility data indicated that the thermodynamic stability rank order from highest to lowest is A04 followed by A03, A02 and then A01 at ambient temperature. As A05 immediately transformed to A04 in the solvent, determination of its solubility was not possible. However, A05 was found to be less stable than A01 using DSC. The overall rank order is therefore A04 followed by A03, A02, A01, and then A05.

Proposed Method for Estimating Transition Temperatures with Solution Calorimetry Polymorphic transition temperatures can be estimated using solution calorimetry. ${ }^{12,13)}$
This method is effective when a limited quantity of sample is available. The transition temperatures from A02 to A01 and A03 to A04 were therefore estimated using this method.

When solubility of two polymorphs (A and B) is expressed using the van't Hoff equation, the solubility of each polymorph is presented by Eqs. 1 and 2, as follows:

$$
\begin{aligned}
& \ln x_{\mathrm{A}}=\left(-\Delta H_{\mathrm{soln}, \mathrm{A}} / R\right) T^{-1}+C_{1} \\
& \ln x_{\mathrm{B}}=\left(-\Delta H_{\mathrm{soln}, \mathrm{B}} / R\right) T^{-1}+C_{2}
\end{aligned}
$$

where, $x$ denotes the mole fraction solubility, $\Delta H_{\text {soln }}$ denotes the heat of solution, $R$ denotes the gas constant, $T$ denotes absolute temperature, and $C$ denotes a constant. At temperatures $T_{1}$ and $T_{2}$, subtracting Eq. 2 from Eq. 1 gives Eqs. 3 and 4, as follows:

$$
\begin{aligned}
& \ln x_{\mathrm{A}, T_{1}}-\ln x_{\mathrm{B}, T_{1}}=\left(-\Delta H_{\text {trans }, T_{1}} / R\right) T_{1}^{-1}+\left(C_{1}-C_{2}\right) \\
& \ln x_{\mathrm{A}, T_{2}}-\ln x_{\mathrm{B}, T_{2}}=\left(-\Delta H_{\text {trans }, T_{2}} / R\right) T_{2}^{-1}+\left(C_{1}-C_{2}\right)
\end{aligned}
$$

where, $\Delta H_{\text {trans, } T}$ is the heat of transition $\left(\Delta H_{\text {soln }, \mathrm{A}}-\Delta H_{\mathrm{soln}, \mathrm{B}}\right)$ at T. Subtracting Eq. 4 from Eq. 3 gives Eq. 5, as follows:

$$
\begin{aligned}
& \left(\ln x_{\mathrm{A}, T_{1}}-\ln x_{\mathrm{B}, T_{1}}\right)-\left(\ln x_{\mathrm{A}, T_{2}}-\ln x_{\mathrm{B}, T_{2}}\right) \\
& \quad=\left(-\Delta H_{\text {trans }, T_{1}} / R\right) T_{1}^{-1}-\left(-\Delta H_{\text {trans }, T_{2}} / R\right) T_{2}^{-1}
\end{aligned}
$$

When the heat of solution is considered independent of temperature in a narrow temperature range between $T_{1}$ and $T_{2}$, $\Delta H_{\text {trans, } T_{1}}$ and $\Delta H_{\text {trans, } T_{2}}$ is considered equal. When $T_{2}$ is $T_{\text {trans }}$, $\ln x_{\mathrm{A}, T_{2}}-\ln x_{\mathrm{B}, T_{2}}$ is zero. Therefore, Eq. 5 becomes Eq. 6, as follows:

$$
\ln x_{\mathrm{A}, T_{1}}-\ln x_{\mathrm{B}, T_{1}}=\left(-\Delta H_{\text {trans }} / R\right)\left(T_{1}^{-1}-T_{\text {trans }}^{-1}\right)
$$

Equation 6 indicates that when $\Delta H_{\text {trans }}$ and solubility at one temperature are already known, $T_{\text {trans }}$ can be estimated. The heat of solution of each polymorph in methyl ethyl ketone was measured using a solution calorimeter (TAM III; TA Instruments) to calculate the transition temperature. 
Table 2. Transition Temperature Estimated Using a Solution Calorimetry

\begin{tabular}{|c|c|c|c|c|c|c|}
\hline Crystal form & $\begin{array}{l}\text { Heat of dissolution } \\
\qquad[\mathrm{kJ} / \mathrm{mol}]\end{array}$ & $\begin{array}{c}\text { Solubility in methyl } \\
\text { ethyl ketone }[\mathrm{mol} / \mathrm{mol}]\end{array}$ & $T_{1}[\mathrm{~K}]$ & $\Delta H_{\text {trans }}[\mathrm{kJ} / \mathrm{mol}]$ & $T_{\text {trans }}($ Eq. 6) $[\mathrm{K}]$ & $\begin{array}{c}T_{\text {trans }} \text { (van't Hoff plots) } \\
{[\mathrm{K}]}\end{array}$ \\
\hline $\mathrm{A} 01$ & 25.01 & 0.00221 & 298 & 1.88 & 326 & 325 \\
\hline A02 & 26.89 & 0.00207 & & & & \\
\hline $\mathrm{A} 03$ & 32.25 & 0.00109 & 298 & 0.61 & 1498 & 1400 \\
\hline A04 & 32.86 & 0.00089 & & & & \\
\hline
\end{tabular}

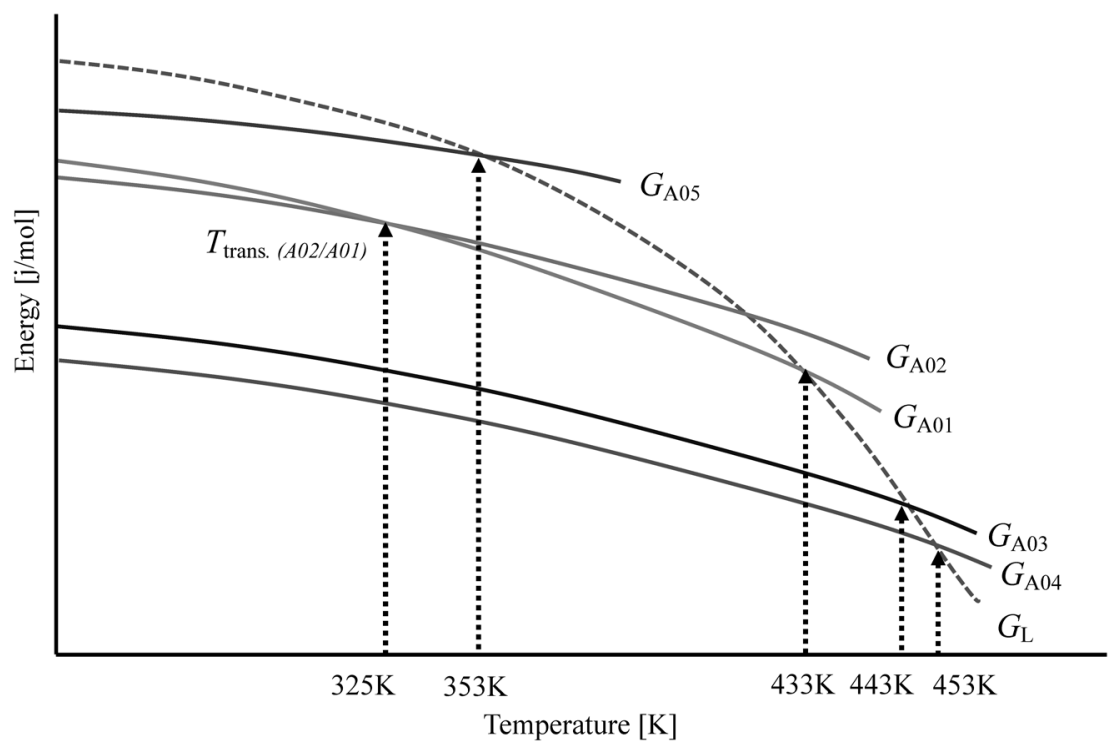

Fig. 6. Temperature Energy Diagram

The transition temperatures estimated by Eq. 6 are shown in Table 2. The transition temperature from A02 to A01 was $326 \mathrm{~K}$, which was nearly equal to the temperature estimated by the van't Hoff plot $(325 \mathrm{~K})$. Further, the transition temperature from A04 to A03 can be estimated by this solution calorimetry method $(1498 \mathrm{~K})$, which is again consistent with the result obtained using the van't Hoff plot (1400 K).

The schematic diagram showing the interrelation of stability among the polymorphs is illustrated in Fig. 6 .

\section{Conclusion}

The thermodynamic stability of each ASP3026 polymorph was investigated in detail, and the stability of each was clarified by DSC analysis or a van't Hoff plot. A04 was the most stable at temperatures below the melting point. The relationship between A04 and A03 and A04 and A01 were mutually monotropic, while that between A01 and A02 was enantiotropic. The transition temperature from A02 to A01 was estimated as $325 \mathrm{~K}$. A02 was thermodynamically more stable than A01 at ambient temperature, while A05 was the least stable polymorph. Solution calorimetry was effective for the estimation of polymorphic transition temperatures. This method might be particularly effective during the early stage of development when a limited quantity of sample is available. The systematic characterization of ASP3026 polymorphs presented in this study was effective for the selective crystallization of the most stable polymorph and the design of solid formulations.
Conflict of Interest Kazuhiro Takeguchi, Yutaka Hirakura, Kouji Yamazaki, Itsuro Shimada, Shigeru Ieda, and Minoru Okada are employees of Astellas Pharma Inc.

\section{References}

1) Kondoh Y., Hkubo K., Kuromitsu S., Shindo N., Soga T., Furutani T., Shimada I., Matsuya T., Kurosawa K., Kamikawa A., Mano H., PCT Int. Appl., WO2009008371 A1 (2009).

2) Shimada I., Hirakura Y., Yamazaki K., Takeguchi K., Ueda N., PCT Int. Appl., WO2011145548 A1 (2011).

3) Datta S., Grant D., Nat. Rev. Drug Discov., 3, $42-57$ (2004).

4) Borka L., Haleblian J., Act. Pharm. Jigosl., 40, 71-94 (1990).

5) Haleblian J., McCrone W., J. Pharm. Sci., 58, 911-929 (1969).

6) Singhal D., Curatolo W., Adv. Drug Deliv. Rev., 56, 335-347 (2004).

7) Kimura N., Fukui H., Takagaki H., Yonemochi E., Terada K., Chem. Pharm. Bull., 49, 1321-1325 (2001).

8) Burger A., Ramberger R., Mikrochim. Acta II (Vienna), 259-271 (1979).

9) Burger A., Ramberger R. Mikrochim. Acta II (Vienna), 273-316 (1979).

10) Chemburkar S. R., Bauer J., Deming K., Spiwek H., Patel K., Morris J., Henry R., Spantpon S., Dziki W., Porter W., Quick J., Bauer P., Donaubauer J., Narayanan B., Soldani M., Riley D., McFarland K., Org. Proc. R\&D, 4, 413-417 (2000).

11) Prashad M., Sutton P., Wu R., Hu B., Vivelo J., Carosi J., Kapa P., Liang J., Org. Proc. R\&D, 14, 878-882 (2010).

12) Urakami K., Shono Y., Higashi A., Umemoto K., Godo M., Chem. Pharm. Bull., 50, 263-267 (2002).

13) Minamisono T., Omae T., Kato T., Takiyama H., J. Chem. Eng. Japan, 44, 197-202 (2011). 